\title{
Automated Glycan Assembly in a Variable-Bed Flow Reactor Provides Insights into Oligosaccharide-Resin Interactions
}

\author{
Eric T. Sletten, José Danglad-Flores, Manuel Nuño, Duncan Guthrie, and Peter H. Seeberger*
}

Cite This: Org. Lett. 2020, 22, 4213-4216

Read Online

ABSTRACT: A pressure-based variable-bed flow reactor built for peptide synthesis and capable of real-time monitoring of resin swelling was adapted for automated glycan assembly. In the context of the solidphase synthesis of several oligosaccharides, the coupling efficiencies, resin growth patterns, and saccharide solvation during the synthesis were determined. The presented work provides the first estimation of on-resin oligosaccharide solvation and an alternative technique to UV-vis monitoring.

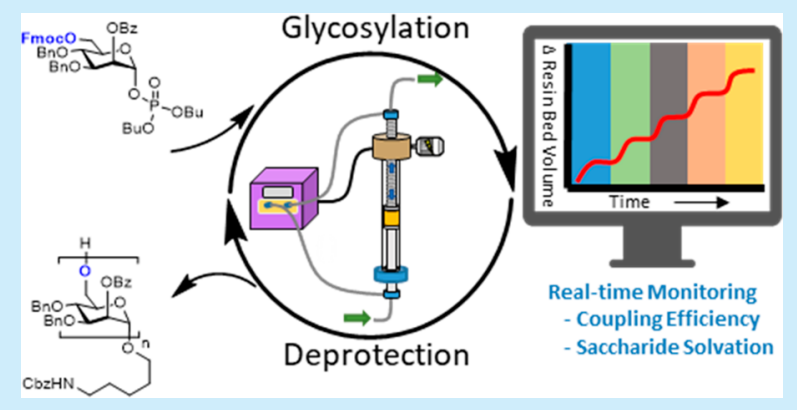

A utomated solid-phase synthesis is a facile way to assemble biopolymers, such as oligonucleotides, peptides, and oligosaccharides. ${ }^{1-3}$ Insights into the interplay of peptide, resin, and solvent have proven fundamental to advancing automated solid-phase peptide synthesis (SPPS). To further improve the efficiency of automated glycan assembly (AGA), insights concerning physicochemical phenomena inside the resin during each reaction cycle are essential. ${ }^{4}$

Reactions involving reagents attached to a solid-phase resin are performed within a swollen cross-linked polymer as the reaction medium. ${ }^{5,6}$ Automated continuous-flow peptide synthesis has benefitted from a variable-bed flow reactor (VBFR) system that allows for the synthesis cycle to be monitored in real time. ${ }^{7-10}$ For that purpose, differential pressure changes across the reaction bed caused by resin swelling and shrinking are measured. The system autonomously adjusts the bed volume to maintain the same differential pressure to allow for the resin to swell freely and avoid reagent channeling. Changes in resin volume correlate with changes in the internal polymer matrix and peptide chain, yet the effect of the growing oligosaccharide on the resin volume is not understood.

To date, monitoring of solid-phase oligosaccharide syntheses has relied on methods such as magic-angle spinning nuclear magnetic resonance (NMR) or other methods that cannot be incorporated into an automated system. ${ }^{11}$ Quantification of the cleaved fluorenylmethoxycarbonyl (Fmoc) temporary protecting group is used for batch and continuous-flow oligosaccharide synthesis. ${ }^{12,13}$ The technique helps to approximate the efficiency of a previous coupling cycle, but it does not allow for synthesis adjustments. Fmoc quantification requires a specific protecting group and provides no insight into the oligosaccharide-resin interactions. ${ }^{12,13}$ In contrast, VBFR monitoring can detect minute resin volume changes due to saccharide incorporation, allowing for coupling efficiencies to be assessed in real-time. Problematic couplings are immediately identified, and adjustments can be made during the synthesis while not being restrained to Fmoc or UV-active protecting groups. The VBFR system was employed to study resin growth patterns and saccharide solvation for each step of the AGA cycle.?

A VBFR was integrated into a fully automated flow system that was adapted for oligosaccharide assembly (Figure 1 and Figure S1). The setup consists of an autosampler and two Vapourtec R-series pump modules for delivery of the reagents. Cooling coils prevent premature activation of the glycosyl donor. A VBFR system autonomously adjusts the resin bed size based on differential pressures. For additional monitoring after the resin bed, an in-line UV-vis detector provides input on whether the reagents were delivered properly. Lastly, the system contains an active back-pressure regulator to ensure a steady flow throughout the synthesis. The Vapourtec Flow Commander software controls the system and records the UV-vis and VBFR chromatograms in real-time.

Initially, conditions suitable for the VBFR flow system and AGA had to be identified. To ensure that resin swelling is a result of resin-bound glycan changes, only one solvent can be used throughout the synthesis. Chloroform proved to be the best choice considering the boundary conditions because it has a relatively lowvapor pressure to avoid cavitation, it is not Lewis basic, it swells the resin well, and it is not aromatic. The

Received: April 10, 2020

Published: May 12, 2020 


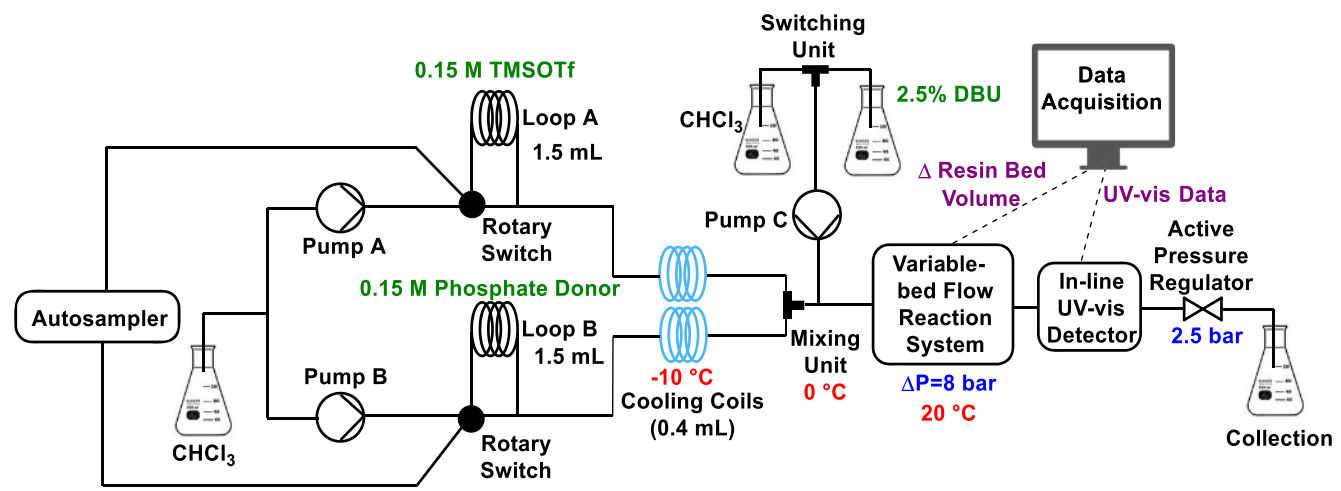

Figure 1. Flow diagram of the AGA-VBFR. For additional photos, see the SI.

first monosaccharide is coupled to the resin by delivering mannosyl phosphate building block 1 (Figure 1, Loop B, 0.15 $\mathrm{M}$ in anhydrous $\mathrm{CHCl}_{3}$ ) and the activator, TMSOTf (Loop A, $0.15 \mathrm{M}$ in anh. $\mathrm{CHCl}_{3}$ ), at a flow rate of $0.3 \mathrm{~mL} \mathrm{~min}^{-1}$. 14,15 The reagents were first pumped through cooling coils at -10 ${ }^{\circ} \mathrm{C}$. Upon mixing at low temperature, the activated building block warmed to room temperature immediately prior to passing to the VBFR system, ensuring that the resin bed was the primary reaction location and avoiding premature decomposition. Throughout the synthesis, the VBFR system maintained a reactor differential pressure of 8 bar (overall system pressure $=10.5$ bar) by adjusting the resin bed volume. Removal of the temporary Fmoc protecting group was achieved using a solution of DBU in chloroform (pump C) instead of piperidine, which is prone to precipitation in chlorinated solvents. ${ }^{16}$

With the preliminary conditions in hand, the VBFR system was used for the final optimization of each step, employing a double coupling cycle, followed by Fmoc removal (Figure S2). A maximum resin volume increase $(\Delta$ resin bed volume $=0.08$ $\mathrm{mL}, 0.1 \mathrm{~g}$ of resin, loading $=0.33 \mathrm{mmol} \mathrm{g}^{-1}$ ) was achieved when 7.25 equiv of building block and activator were used to reach full saturation of the resin during the glycosylation step (blue section, Figure 2). ${ }^{7}$ A $2.5 \%$ DBU solution $(8 \mathrm{~mL}$, flow

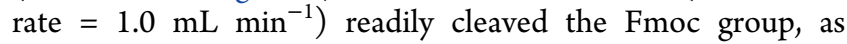
depicted by a loss in the resin bed volume until a new equilibrium volume was reached (red section, Figure 2). The sharp changes in resin bed volume between steps are a result of the flow rate changes causing the differential pressure across the resin bed to be altered. Overall, one synthetic cycle including two wash steps (green and purple sections, Figure 2) produced $\mathrm{C}(6)-\mathrm{OH}$ monosaccharide 2 in $51 \%$ yield in 39 min. Coupling of the monosaccharide to the resin caused a change in the overall resin bed volume that was tracked by the VBFR system. Although unoptimized, these conditions helped to track the glycosylation of pentaacetylated mannose phosphate donor S1 to the resin, highlighting the protecting group flexibility that the VBFR system can monitor (Figure S5).

To determine whether oligosaccharides can be assembled using repetitive automated cycles, an acidic wash of the activator solution ( $0.075 \mathrm{M}$ TMSOTf) was passed over the resin to reduce the buildup of basic DBU. Interestingly, three cycles employing these conditions resulted in $>25 \%$ deletion sequences according to high-performance liquid chromatography (HPLC) analysis. An examination of the UV-vis traces during Fmoc cleavage shows similar peaks for each cycle (Figure S3). However, the VBFR trace helped to resolve the

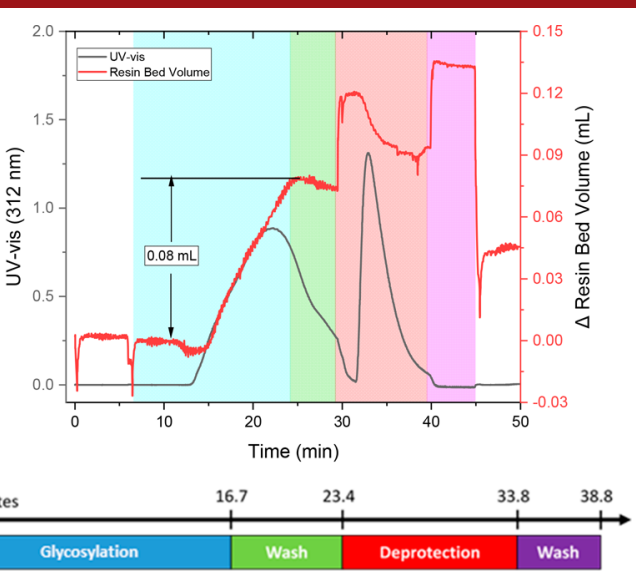

Figure 2. (Top) Read-out of the AGA-VBFR system for a typical coupling cycle. On the right $y$ axis of the AGA-VBFR trace, the graph presents the online accumulative variation of the resin bed volume (red line). The black line displays the time-resolved UV-vis (left $y$ axis, $312 \mathrm{~nm}$ ) absorption of the reactor out stream. (Bottom) A colorcoded timeline matches the corresponding time segments on the graph. Flow rates $\left(\mathrm{mL} \mathrm{min}^{-1}\right)$ : Glycosylation $=0.3$; Wash $\# 1=0.3$; Deprotection $=1.0$; Wash $\# 2=2.0$. For additional details of the experimental conditions, see the SI.

problem because the increase in the resin bed volume returned to the original position prior to glycosylation after the acidic wash, indicating that the attached saccharide was cleaved from the resin under acidic conditions. The cleavage may occur due to the elevated temperature $\left(20^{\circ} \mathrm{C}\right)$ in comparison with other automated systems where the acidic wash is carried out at -20 ${ }^{\circ} \mathrm{C} .{ }^{13}$ Substitution of the acidic wash with an extended chloroform wash (flow rate $=2 \mathrm{~mL} \mathrm{~min}^{-1}$ ) proved sufficient to remove the DBU (Figure 2).

After optimization, the VBFR system was able to track the growth patterns throughout the synthesis of $\alpha(1-6)$-di- (3), tri- (4), and penta-mannosides (5) (0.1 g resin, loading $=0.33$ mmol g $\left.{ }^{-1}\right){ }^{17}$ Oligosaccharides $3-5$ were produced in yields that are consistent with the even resin growth patterns (Figure $3, \Delta$ volume $=+0.080 \mathrm{~mL} /$ coupling and $-0.035 \mathrm{~mL} /$ Fmoc removal). Pentamannoside 5 was prepared in $3.5 \mathrm{~h}$ using the AGA-VBFR system and consumed only $27 \mathrm{~mL}$ of solvent per cycle. ${ }^{12,18}$ Attempts using other building blocks under these conditions resulted in deletion sequences likely because the reaction temperature, flow rate, and amount of activator need to be adjusted.

The coupling of a monosaccharide building block to the resin causes the resin bead to expand beyond that of the 


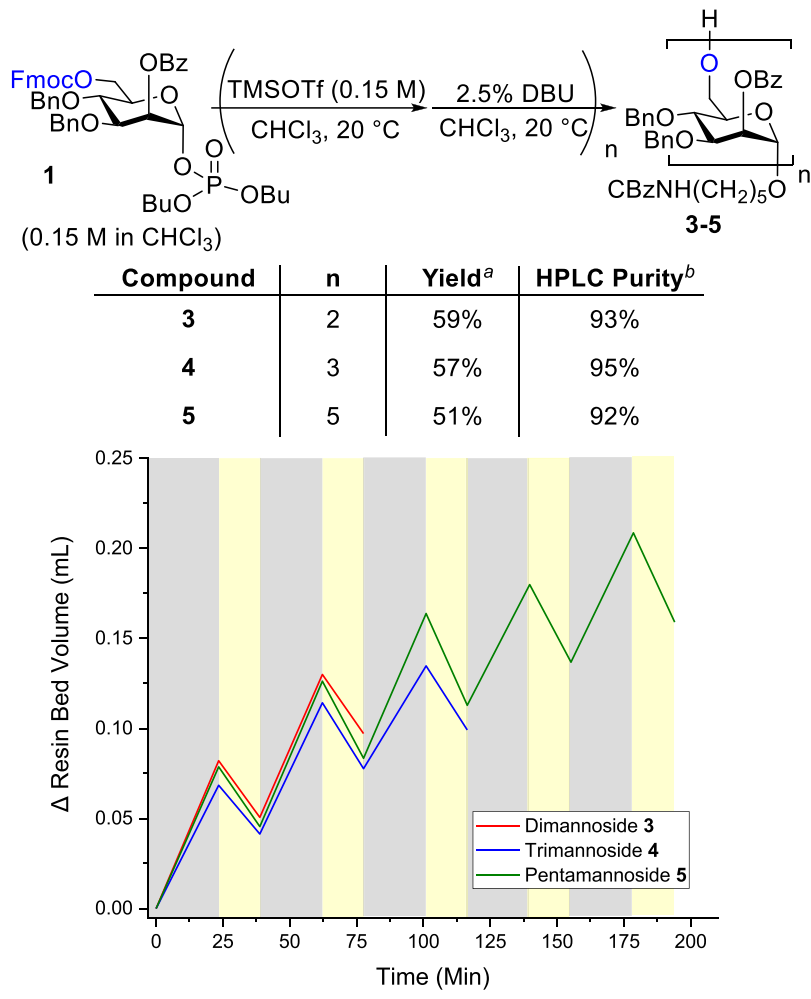

Figure 3. AGA-VBFR synthesis of oligomannosides 2-5. (Top) Optimized conditions and yields for the synthesis of oligomannosides 3-5. (Bottom) Change in the resin bed volume after each cycle with respect to process time $($ gray $=$ glycosylation, yellow $=$ Fmoc removal). Each line corresponds to one oligosaccharide synthesized. For raw traces, see the Supporting Information. ${ }^{a}$ Isolated yield. ${ }^{b}$ See the Supporting Information for full HPLC traces.

volume of the initial unsubstituted swollen resin. Regressive analysis of the change in the resin bed volume after each deprotection cycle has a quadratic tendency $\left(R^{2}=0.999\right)$. The volume change is large for the first coupling; then, as the oligosaccharide chain grows, the intrinsic elastic restraining force of the cross-linked resin begins to lessen the volume change (Figure $4 a$, red line). ${ }^{6,10}$ The resin growth pattern of oligosaccharides is similar to that of a nonaggregating peptide (sequence $=$ AFLAFLA, Figure $4 \mathrm{a}$, blue line) in DMF. ${ }^{7}$ The resin growth for three to four amino acids equals the volume gained by the addition of one mannose unit.

In addition to the size of a molecule, the solvent and solvation also play a vital role in the resin volume change. ${ }^{6,19}$ Polystyrene resin will result in enhanced swelling if the interactions with the solvent, such as the hydrogen bonding of dichloromethane or chloroform with the $\pi$ electrons of the aromatic nuclei of polystyrene, are more favorable than selfinteractions between the aromatic systems of the sytrenes. ${ }^{6}$ Similar interactions of the aromatic moieties of the protecting groups on the oligosaccharide make them highly soluble in these solvents.

With the swollen cross-linked polymer acting as the reaction medium, solvation effects control how fast a molecule can enter the resin and the subsequent reaction. The VBFR data and computed saccharide volumes allowed for the calculation (see the SI) of the solvation number of each mannose unit over the course of the pentamannoside synthesis (Figure $4 b$ ). Initially, when the restraining forces of the resin are low, the change in volume comes not just from the incoming a)
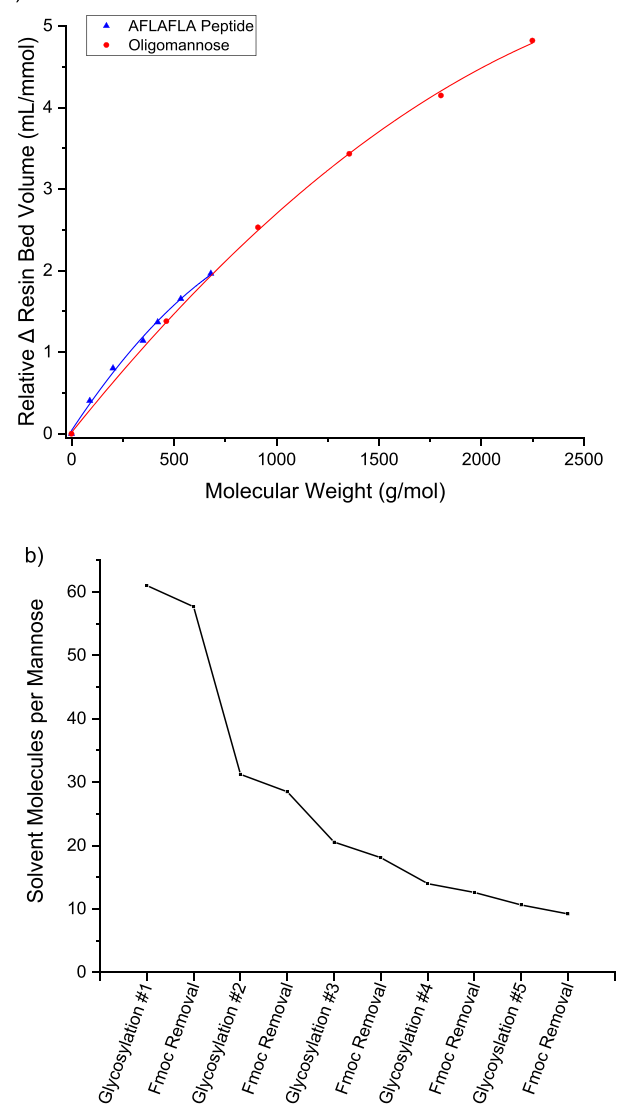

Figure 4. (a) Comparative VBFR growth patterns for an oligosaccharide and a peptide. (b) Solvation number per mannose unit during the synthesis of pentamannoside 5 .

saccharide, but also an additional amount of $10 \mu \mathrm{L}$ of $\mathrm{CHCl}_{3}$ is introduced into the resin beads (Figure S6). After the first glycosylation, the resin contains a total amount of 60 $\mathrm{CHCl}_{3}$ molecules per mannose monosaccharide. As the oligosaccharide elongates, the restraining forces become greater and the oligosaccharide begins to displace solvent molecules from the initial swollen state. Eventually, an equilibrium is reached, and the trend begins to plateau at nine $\mathrm{CHCl}_{3}$ molecules per mannose saccharide unit for pentamannoside 5 (Figure $4 \mathrm{~b}$ ). Per individual unit, pentamannoside 5 is more solvated than peptides (DMF $=1-3$ and $\mathrm{CH}_{2} \mathrm{Cl}_{2}=0.3$ to 1$){ }^{10}$ The inherent difference results from a complementary solubilizing effect, as chloroform provides favorable interactions for both the sugar and resin. In SPPS, dichloromethane is a better swelling solvent than DMF for polystyrene, whereas DMF is the better solvating medium for protected peptides. We believe that the highly solvated state of the growing oligomannoside prevents self-association and onresin aggregation. The swollen state of the oligosaccharideresin provides the freedom for rapid and complete chemical reactions during subsequent couplings. The effect of the protecting groups on the solvation properties of the growing oligosaccharide have yet to be understood, as they contribute greatly to the overall polarity of the molecule and will be the subject of subsequent studies.

The incorporation of a VBFR system into an automated oligosaccharide synthesizer provided a platform to efficiently synthesize several oligosaccharides while efficiently monitoring each step in real time without constraint to a particular 
protecting group. The VBFR system tracks the resin growth pattern during AGA, which can be translated into the solvation state of each monosaccharide and cannot be obtained by any other monitoring system. These proof-of-concept studies concerning the physiochemical resin-oligosaccharide properties provide a tool to optimize solid-phase oligosaccharide synthesis.

\section{ASSOCIATED CONTENT}

\section{Supporting Information}

The Supporting Information is available free of charge at https://pubs.acs.org/doi/10.1021/acs.orglett.0c01264.

Experimental procedures and characterization data (PDF)

\section{AUTHOR INFORMATION}

\section{Corresponding Author}

Peter H. Seeberger - Department of Biomolecular Systems, Max Planck Institute of Colloids and Interfaces, 14476 Potsdam, Germany; Department of Chemistry and Biochemistry, Freie Universität Berlin, 14195 Berlin, Germany; (1) orcid.org/0000-0003-3394-8466;

Email: peter.seeberger@mpikg.mpg.de

\section{Authors}

Eric T. Sletten - Department of Biomolecular Systems, Max Planck Institute of Colloids and Interfaces, 14476 Potsdam, Germany

José Danglad-Flores - Department of Biomolecular Systems, Max Planck Institute of Colloids and Interfaces, 14476 Potsdam, Germany

Manuel Nuño - Vapourtec, Ltd., Bury St. Edmunds IP28 6TS, United Kingdom

Duncan Guthrie - Vapourtec, Ltd., Bury St. Edmunds IP28 6TS, United Kingdom

Complete contact information is available at:

https://pubs.acs.org/10.1021/acs.orglett.0c01264

\section{Author Contributions}

E.T.S. and P.H.S. designed the experiments that E.T.S. and J.D.-F. performed. E.T.S. and P.H.S. wrote the manuscript. D.G. developed the continuous-flow VBFR. M.N. trained E.T.S. to use the system. D.G. and M.N. loaned the prototype instrument for the duration of the research.

Notes

The authors declare no competing financial interest.

\section{ACKNOWLEDGMENTS}

We thank the Max Planck Society for the generous financial support. We are grateful to Theodore Tyrikos-Ergas, Mara Guidi, Dr. Mónica Guberman, Dr. Kim Le Mai Hoang, Dr. Bartholomäus Pieber, and Dr. Martina Delbianco for providing insightful advice. We also thank Sabrina Leichnitz for help with recording the HRMS. All of the acknowledged work was performed at the Max Planck Institute of Colloids and Interfaces.

\section{REFERENCES}

(1) Merrifield, R. B.; Stewart, J. M.; Jernberg, N. Instrument for automated synthesis of peptides. Anal. Chem. 1966, 38, 1905-1914.
(2) Alvarado-Urbina, G.; Sathe, G. M.; Liu, W. C.; Gillen, M. F.; Duck, P. D.; Bender, R.; Ogilvie, K. K. Automated synthesis of gene fragments. Science 1981, 214, 270.

(3) Plante, O. J.; Palmacci, E. R.; Seeberger, P. H. Automated SolidPhase Synthesis of Oligosaccharides. Science 2001, 291, 1523.

(4) Vaino, A. R.; Janda, K. D. Solid-Phase Organic Synthesis: A Critical Understanding of the Resin. J. Comb. Chem. 2000, 2, 579596.

(5) Czarnik, A. W. Solid-phase synthesis supports are like solvents. Biotechnol. Bioeng. 1998, 61, 77-79.

(6) Sarin, V. K.; Kent, S. B. H.; Merrifield, R. B. Properties of swollen polymer networks. Solvation and swelling of peptidecontaining resins in solid-phase peptide synthesis. J. Am. Chem. Soc. 1980, 102, 5463-5470.

(7) Sletten, E. T.; Nuño, M.; Guthrie, D.; Seeberger, P. H. Real-time monitoring of solid-phase peptide synthesis using a variable bed flow reactor. Chem. Commun. 2019, 55, 14598-14601.

(8) Baru, M. B.; Mustaeva, L. G.; Vagenina, I. V.; Gorbunova, E. Y.; Cherskii, V. V. Pressure monitoring of continuous-flow solid-phase peptide synthesis. J. Pept. Res. 2001, 57, 193-202.

(9) Rodionov, I.; Baru, M. B.; Ivanov, T. E. A swellographic approach to monitoring continuous flow solid phase peptide synthesis. Pept. Res. 1992, 5, 119-125.

(10) Rodionov, I. L.; Peshenko, I. A.; Baidakova, L. K.; Ivanov, V. T. Swellographic Study of Peptide Resin Swelling Behavior during Solid Phase Peptide Synthesis. Int. J. Pept. Res. Ther. 2007, 13, 161-171.

(11) Bennett, C. S. Principles of modern solid-phase oligosaccharide synthesis. Org. Biomol. Chem. 2014, 12, 1686-1698.

(12) Ganesh, N. V.; Fujikawa, K.; Tan, Y. H.; Stine, K. J.; Demchenko, A. V. HPLC-Assisted Automated Oligosaccharide Synthesis. Org. Lett. 2012, 14, 3036-3039.

(13) Hahm, H. S.; Schlegel, M. K.; Hurevich, M.; Eller, S.; Schuhmacher, F.; Hofmann, J.; Pagel, K.; Seeberger, P. H. Automated glycan assembly using the Glyconeer 2.1 synthesizer. Proc. Natl. Acad. Sci. U. S. A. 2017, 114, E3385.

(14) Plante, O. J.; Andrade, R. B.; Seeberger, P. H. Synthesis and Use of Glycosyl Phosphates as Glycosyl Donors. Org. Lett. 1999, 1, 211-214.

(15) Plante, O. J.; Palmacci, E. R.; Andrade, R. B.; Seeberger, P. H. Oligosaccharide Synthesis with Glycosyl Phosphate and Dithiophosphate Triesters as Glycosylating Agents. J. Am. Chem. Soc. 2001, 123, 9545-9554.

(16) Yang, Y. Chapter 14 - Solvent-Induced Side Reactions in Peptide Synthesis. In Side Reactions in Peptide Synthesis; Yang, Y., Ed.; Academic Press: Oxford, U.K., 2016; pp 311-322.

(17) Eller, S.; Collot, M.; Yin, J.; Hahm, H. S.; Seeberger, P. H. Automated Solid-Phase Synthesis of Chondroitin Sulfate Glycosaminoglycans. Angew. Chem., Int. Ed. 2013, 52, 5858-5861.

(18) Delbianco, M.; Kononov, A.; Poveda, A.; Yu, Y.; Diercks, T.; Jiménez-Barbero, J.; Seeberger, P. H. Well-Defined Oligo- and Polysaccharides as Ideal Probes for Structural Studies. J. Am. Chem. Soc. 2018, 140, 5421-5426.

(19) Fields, G. B.; Fields, C. G. Solvation effects in solid-phase peptide synthesis. J. Am. Chem. Soc. 1991, 113, 4202-4207. 